EOMmUn Communication et organisation

Ory

Structure et communication

\title{
La communication à Casablanca
}

Hassan Esmili

\section{OpenEdition}

Journals

Édition électronique

URL : http://journals.openedition.org/communicationorganisation/1604

DOI : 10.4000/communicationorganisation. 1604

ISSN : 1775-3546

\section{Éditeur}

Presses universitaires de Bordeaux

\section{Édition imprimée}

Date de publication : 1 novembre 1992

ISSN : 1168-5549

\section{Référence électronique}

Hassan Esmili, «La communication à Casablanca », Communication et organisation [En ligne], 2 | 1992, mis en ligne le 26 mars 2012, consulté le 19 avril 2019. URL : http://journals.openedition.org/ communicationorganisation/1604 ; DOI : 10.4000/communicationorganisation.1604

Ce document a été généré automatiquement le 19 avril 2019

(C) Presses universitaires de Bordeaux 


\title{
La communication à Casablanca
}

\author{
Hassan Esmili
}

1 La faculté des lettres et des sciences humaines de Benmsik à Casablanca vient de créer une licence (l'équivalent d'une maîtrise française) en communication et une autre en arts appliqués. Pour comprendre l'importance et l'intérêt de cette création, il est nécessaire de l'insérer dans le contexte global de l'enseignement supérieur au Maroc.

2 Il y a dans ce pays, au niveau de l'enseignement supérieur public, qui est sous la tutelle du ministère de l'éducation nationale, cinq sortes d'établissements :

- les écoles supérieures de technologie qui admettent sur concours les titulaires du baccalauréat, série mathématiques, et qui donnent une formation de technicien supérieur en deux années ;

- les écoles d'ingénieurs qui admettent sur concours les élèves bacheliers, série mathématiques, qui ont réussi les deux années des classes préparatoires ; ils y reçoivent une formation d'ingénieur de conception en trois ans ;

- les facultés de médecine, de médecine dentaire et de pharmacie qui admettent sur concours les bacheliers des séries mathématiques et sciences expérimentales; elles donnent une formation de cinq ans pour les chirurgiens dentistes et les pharmaciens et une formation de sept ans pour les médecins généralistes ; le temps de formation des spécialistes varie de trois à quatre ans selon les spécialités ;

- les facultés des sciences qui admettent, sans concours, les bacheliers des séries mathématiques et sciences expérimentales et qui préparent à des licences en quatre années et à des doctorats ;

- les facultés des lettres et des sciences humaines et les facultés des sciences juridiques, économiques et sociales qui admettent, sans concours, les bacheliers de toutes les séries et qui préparent à des licences en quatre années et à des doctorats.

On peut remarquer à partir de cette énumération qu'il existe des établissements qui préparent directement à une profession et qui pratiquent une forte sélection dés l'accès ; et d'autres qui offrent des formations générales, qui sont libres d'accès (sauf pour les sciences où l'on regarde la nature du baccalauréat).

4 Les facultés des lettres se trouvent dans cette dernière catégorie; tous les étudiants refoulés des autres établissements peuvent venir réclamer le droit de s'inscrire dans une 
faculté de droit ou de lettres à côté des pauvres bacheliers de la série «lettres modernes ». Il est évident que cette situation rejaillit sur la qualité des formations et sur l'image de marque de ces établissements, qui est peu prestigieuse.

5 Ceci nous a conduit depuis quelques années à réfléchir, avec des collègues, è une nouvelle conception de la faculté des lettres et des sciences humaines fondée sur les principes suivants :

6 1. Atténuer l'esprit académique en portant plus d'intérêt aux questions actuelles, en s'ouvrant sur l'environnement et en repensant les méthodes pédagogiques.

7 2. Cuvrer pour une meilleure adéquation entre les formations et les domaines d'activité ciblés, sans pour autant transformer la faculté en une école professionnelle.

8 3. Faire de l'espace facultaire un espace d'expression et de création.

9 4. Contribuer au développement et au rayonnement culturels par l'encouragement et le soutien des potentialités estudiantines.

10 5. Encourager la recherche fondamentale et la recherche-action (ou appliquée), la structurer et développer l'esprit d'équipe.

11 C'est dans ce cadre que nous avons placé toute l'action conduite è la faculté de Benmsik depuis sa création en 1984.

12 Les ateliers ont ouvert aux étudiants la possibilité de travailler dès 85 dans des domaines comme l'écriture et la traduction, la vidéo, l'analyse filmique, la musique et le chant, les arts plastiques et le théâtre. Plusieurs manifestations nationales et internationales sont nées à partir des ateliers, dont la plus célèbre est le Festival International de Théâtre Universitaire (FTUC) qui se tient tous les ans et qui est devenu une véritable institution.

Des groupes de recherche se sont formes; certains ont décroché des contrats de recherche avec des collectivités ou avec des entreprises. Leurs travaux ont été présentés dans des colloques et publiés.

De la même façon, les départements ont commencé a réfléchir à de nouveaux cursus de formation qui répondraient aux principes 1 et 2 . Les deux licences appliquées retenues cette année sont donc les premiers fruits de cette réflexion et notre faculté a été la première à les proposer et les créer. C'est de la que vient toute l'importance qu'elles revêtent pour nous. La qualité de la formation, le savoir-faire que les étudiants auront acquis seront déterminants dans l'avenir de l'image de marqué de l'institution et des lauréats. Pour avoir le maximum de garanties dans ce sens, nous avons obtenu que ces licences soient sélectives au niveau du recrutement, qu'elles soient à petits effectifs pour la première promotion : 22 en communication et 16 en arts appliqués), et qu'elles soient enfin è aspect pratique. Le diplôme requis dans les deux cas est le DEUG associé à une lettre de motivation et un entretien.

Nous développerons dans cette présentation uniquement le cursus de la licence « communication ».

16 La création de cette spécialité à la faculté répond à un besoin pressenti dans différents types d'organisations ou d'organismes, et notamment dans les entreprises et les collectivités. Il y a une grande carence au niveau des cadres spécialisés à même de s'occuper de la gestion des ressources humaines, de concevoir et de mener une action de communication sociale, ou encore de mettre en place et de gérer un plan de communication interne et/ou externe d'une entreprise. 
17 L'objectif est donc de donner en deux ans une formation intensive et hautement spécialisée fondée sur un apport théorique consistant et un savoir-faire acquis dans des ateliers d'apprentissage pratique et des stages professionnels. Au niveau des méthodes pédagogiques, nous aspirons à créer l'esprit d'autonomie chez l'étudiant et à développer chez lui les mécanismes de la curiosité intellectuelle, de l'observation, de la recherche et de l'auto-apprentissage.

Nous avons quatre types de formateurs qui assurent l'encadrement de cette licence : des enseignants de la faculté recrutés spécialement à cet effet, des enseignants des départements de langues qui ont choisi de se recycler dans la communication, des collègues de l'Institut des Sciences de l'Information et de la Communication de l'Université de Bordeaux 3 avec qui nous avons des liens d'échanges et de coopération depuis quelques années et qui vont animer des séminaires à Casablanca et recevoir des enseignants de la faculté è Bordeaux, et des enseignants vacataires qui viennent d'autres facultés pour donner des cours techniques sur le management ou le marketing par exemple.

En outre, nous faisons appel aux professionnels pour intervenir dans les ateliers et animer les stages en collaboration avec les enseignants.

$20 \mathrm{Au}$ niveau des programmes, nous dispensons dans le cadre de cette licence un enseignement intégré sous forme de blocs. Un premier bloc porte sur les formes et théories de la communication avec un volet sur l'approche théorique qui souligne l'importance de la contextualisation dans la compréhension des phénomènes et l'intérêt de la démarche systémique; un autre volet sur la communication dans les différents contextes sociaux ainsi que les différentes formes de la communication non verbale en relation avec les cultures ; et un troisième sur le rôle de l'espace dans la communication.

21 Le pendant pratique de ce bloc comprend trois unités: la première concerne la méthodologie du travail intellectuel et vise à donner aux étudiants une méthodologie de travail par l'autoformation; la seconde traite de la méthodologie du plan de communication et la troisième unité de la conduite et gestion de projet. Ces deux dernières unités visent à développer les aptitudes des étudiants à appliquer sur le terrain les connaissances théoriques et méthodologiques acquises dans les différents cours, à les synthétiser et à mener de bout en bout un plan de communication commandité. Ils apprendront également à identifier un champ pertinent de ce projet, concevoir ce projet et réaliser un document de présentation, définir les différentes phases de préparation et d'exécution, évaluer le résultat final et le placer dans un processus itératif.

Le second bloc propose un enseignement de psychologie et de sociologie dans une optique communicationnelle. Les étudiants connaitront ainsi les mécanismes psychiques et psycho-physiologiques de l'individu aussi bien que les différents phénomènes de groupe et leurs interactions. Plusieurs aspects sociologiques seront étudiés dans le cadre de la sociologie de la communication, des médias et des pratiques culturelles, de la sociologie des organisations et des masses, et enfin de la sociologie urbaine et rurale. Le corrélat pratique de ce bloc cherche à permettre aux étudiants de maitriser les outils méthodologiques d'investigation telles que les techniques d'enquête, d'entretien, de conduite de réunion d'information ascendante, de marketing. Il leur permettra aussi de pratiquer les techniques d'analyse de contenu, de discours et de pratique, de sémiologie appliquée à l'image et d'observation participante. D'autres ateliers dans le même bloc 
auront à développer chez l'étudiant les aptitudes à la conception et à la création et à le familiariser avec les différentes techniques et méthodes de la production.

Deux autres blocs importants entrent dans cet enseignement. L'un concerne la culture générale avec un cours sur l'histoire du Maroc, d'un point de vue culturel, un cours sur les formes d'expression artistiques au Maroc et enfin un cours d'épistémologie. L'autre bloc concerne des domaines juridiques et économiques en rapport avec le fonctionnement des organisations et des entreprises. On y trouve le cadre juridique de l'information et de la communication, la relation droit et société, la connaissance des principes de base de l'économie de marché avec des applications à l'information, à la communication et au produit culturel, des éléments de gestion des entreprises, les théories, méthodes et techniques du marketing, des éléments de base des statistiques et mathématiques appliquées aux sciences sociales et enfin des éléments de comptabilité.

Voilà en gros comment se présente cette licence appliquée à la communication. C'est une première à l'Université marocaine et une première expérience pour nous à la faculté des lettres et des sciences humaines. C'est un projet conçu par une équipe mixte d'enseignants de Benmsik à Casablanca et de collègues de l'ISIC de Bordeaux 3.

Le feed-back que nous aurons de la part des étudiants, des professionnels et du terrain nous aidera à évaluer cette formation, à la réajuster le cas échéant et l'adapter autant que possible aux différentes attentes.

Espérons que d'autres expériences verront le jour dans les lettres et les sciences humaines et contribueront à la construction d'une nouvelle image de ce domaine du savoir, de l'expression et de la création.

\section{RÉSUMÉS}

La Communication en tant que discipline pratiquée à l'Université marocaine vient de commencer à la rentrée 92. Une licence (maîtrise française) a été créée en collaboration avec l'I.S.I.C. de Bordeaux 3. Hassan Esmili décrit le projet et explique dans quel contexte il a été mis sur pied et quelle importance il représente pour la faculté de lettres. Il montre également que la création de cette nouvelle licence entre dans une politique générale d'adaptation de la formation universitaire au marché de l'emploi.

\section{AUTEUR}

\section{HASSAN ESMILI}

Hassan Esmili est né à Casablanca - Maroc le 4 mai 1946. Après des études primaires et secondaires dans la même ville, il a préparé une licence de langue et littérature françaises à l'Université de Rabat en 1968. Un séjour de quelques années à Paris lui a permis de faire des études de linguistique générale et comparée qui ont abouti à un doctorat en 1977. 
Auteur de plusieurs articles spécialisés, il a enseigné aux universités de Rabat, Fès et Casablanca et a été en (1983) professeur associé à l'Université d'Aix en Provence avant d'être nommé doyen de la Faculté de Lettres de Casablanca Ben M'Sik. 